IMECE2014-39051

\title{
NUMERICAL MODELING OF WAVE SOLDERING IN PCB
}

\author{
Bruno Arcipreste \\ School of Engineering \\ University of Minho \\ 4800-058 Guimarães Portugal
}

\author{
Luis Ribas \\ Bosch Car Multimedia \\ Apartado 2458 \\ Braga Portugal
}

\author{
Delfim Soares \\ School of Engineering \\ University of Minho \\ 4800-058 Guimarães Portugal
}

\author{
José Carlos Teixeira \\ School of Engineering \\ University of Minho \\ 4800-058 Guimarães Portugal
}

\begin{abstract}
Manufacturing of electronic boards (commonly referred as $\mathrm{PCB})$ is a highly automated process that requires an accurate control of the various processing variables. Amongst the soldering processes, wave soldering is one of the most often used. In this, the various electronic components are provisionally inserted onto the PCB, and a low velocity jet of melted solder is directed to the moving board. Due to capillarity effects the solder adheres to the component/board interface and the process is completed. This methodology is most often used for small components. The adjusting of the operating parameters (solder nozzle orientation and velocity) is often carried out on a trial and error basis resulting in a time consuming process that is at odds with the increasing demand for smaller production series that the electronics industry is faced with.

In addition the number of defects (mostly from missing components that are washed away by the impacting jet) is more likely to occur when thinner substrates are used in the PCB manufacturing.

The present paper describes the application of a Computational Fluid Dynamics model to describe the interaction of the solder jet with the PCB and the integrated circuits. The model includes the conservation equations for mass, momentum and energy in a transient time frame. The jet and surrounding ambient atmosphere are modeled as two separate fluids and the interface is tracked by a VOF model. By adjusting the computational mesh refinement the interface is captured with accuracy. The drag forces occurring in the various components are computed from the pressure data field. The model allows the
\end{abstract}

optimization of the wave operating parameters as a function of the component type of and its layout in the PCB.

Keywords: Jet, Cross Flow, CFD.

\section{INTRODUCTION}

Soldering of electronic components onto the Printed Circuit Board (PCB) is the most important process in the electronics industry. Amongst the various soldering processes, wave soldering is used to assemble the components on the PCB $[1,2,3,4]$. This process requires that the various components are temporarily fixed to the PCB in order to support the forces resulting from the wave interaction. Depending on the size of the components, these are fixed by various spots of an adhesive bond on the PCB surface. This holds the component onto the surface before entering the soldering furnace. In this, a conveyor carries the PCB with pin through-hole (PTH) components through the pre-heat, fluxing zone, wave soldering, and cooling zones.

In the wave soldering zone, the PCB with PTH components experience high-temperature molten solder. The bottom surface of the PCB with PTH components interacts with the fountain of the molten solder while the molten solder fills the PCB hole, which is driven by the capillary force of the PCB hole. Subsequently, the filled PCB hole is solidified under the cooling zone. In this process, due to the different heat expansion coefficients in the various materials, the PCB as a whole will experience varying expansion rates that will in thermal stresses and fatigue $[5,6]$. 
One critical aspect concerns the interaction of the molten solder with the PCB surface and the attached components. Due to inadequate dispensing of the amount of adhesive or to the offset of the component to the adhesive spot, some components may detach from the surface before the soldering is complete.

An analysis has been carried out in order to understand the nature of inappropriate binding between the component and the PCB. Most of the deficiencies are related with misalignments in the location of the adhesive spots, as illustrated in Figure 1.

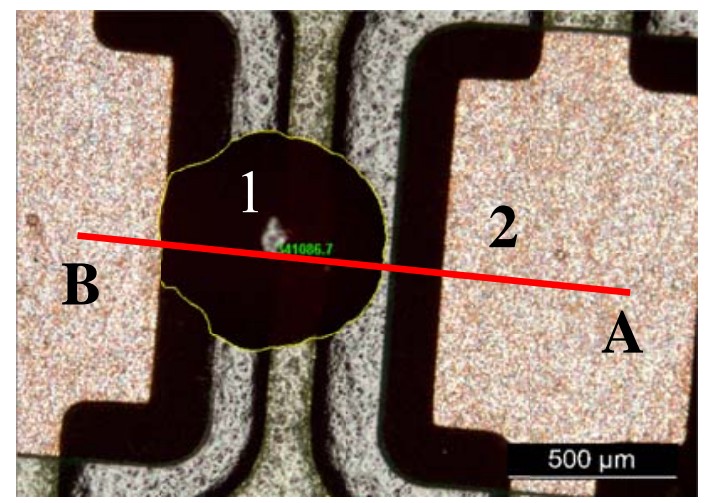

Fig. 1: Misalignment of the adhesive (1) spot relatively to copper pad (2) on the PCB.

A side view clearly along the plane A-B (Figure 1) shows the inappropriate centering of the adhesive (Figure 2).

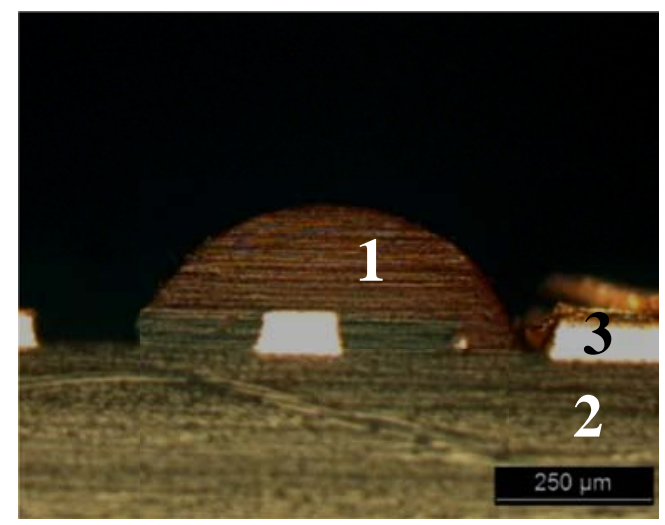

Fig. 2: Cross view of the misalignment of the adhesive spot (1) relatively to copper pad (3) on the PCB (2).

The results show that the number of misaligned adhesive spots is very large for various kinds of components that are typically processed. In addition, when the component is pressed onto the adhesive, other defects can arise from the inclusion of gas bubbles at the interface between the various surfaces. Figure 3 illustrates the presence of unwanted gas bubbles in the interface that yield a reduction of the actual contact area between the component and the PCB. The area reduction can be up to $1 / 4$ of the total contact area.

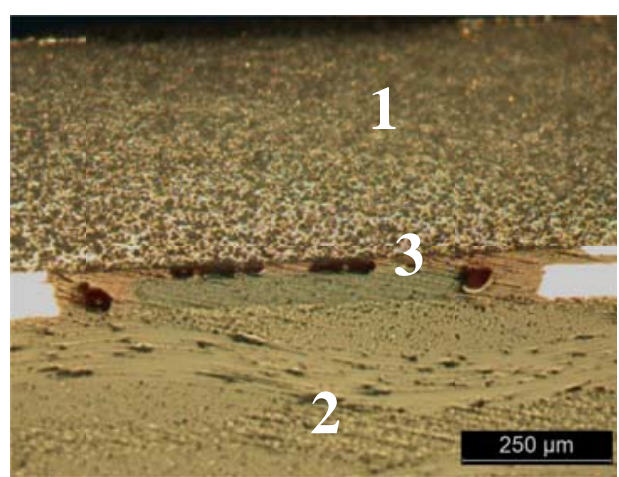

Fig. 3: Presence of gas bubbles (3) between the component (1) and the PCB (2).

The result of such unwanted occurrences is that when the solder wave impacts on the PCB that is processed the adhesion bind may not be strong enough to hold the component.

In addition to improvements in accuracy of the mounting procedure, one may also adjust the characteristics of the wave in order to prevent the parts to be carried over by the solder inertia.

Therefore, understanding the wave soldering process parameters is crucial for a process engineer who aims to achieve high performance of PCB manufacturing process.

The present paper reports the development of a numerical model to describe the formation of the surface wave and its impact upon the PCB surface.

\section{MATHEMATICAL MODEL}

CFD models are a practical, fast and cost effective way to predict fluid behavior in complex situations [7]. Such models include a set of equations that govern the conservation of mass, momentum and energy to compute fluid flow distribution and energy transport in the domain.

Mass conservation implies that the quantity of mass entering a control volume equals the quantity if mass flowing out, creating a balance between input and the output flows for a certain volume. This concept is mathematically expressed by Equation 1, assuming constant the fluid properties.

$$
\frac{\partial u}{\partial x}+\frac{\partial v}{\partial y}+\frac{\partial w}{\partial z}=0
$$

where $u, v$, and $w$ are the velocity in the $x, y$, and $z$ axis. The momentum conservation can be expressed by the rate of change of the momentum of a fluid's particle, and it is equal to the sum of the acting forces at the fluid (Newton's $2^{\text {nd }}$ law). This principle can be described by Equation 2-a through 2-c.

$$
\begin{aligned}
& \rho\left(\frac{\partial u}{\partial t}+u \frac{\partial u}{\partial x}+v \frac{\partial u}{\partial y}+w \frac{\partial u}{\partial z}\right)=-\frac{\partial P}{\partial x}+\eta\left(\frac{\partial^{2} u}{\partial x^{2}}+\frac{\partial^{2} u}{\partial y^{2}}+\frac{\partial^{2} u}{\partial z^{2}}\right)+\rho g_{x} \\
& \rho\left(\frac{\partial v}{\partial t}+u \frac{\partial v}{\partial x}+v \frac{\partial v}{\partial y}+w \frac{\partial v}{\partial z}\right)=-\frac{\partial P}{\partial y}+\eta\left(\frac{\partial^{2} v}{\partial x^{2}}+\frac{\partial^{2} v}{\partial y^{2}}+\frac{\partial^{2} v}{\partial z^{2}}\right)+\rho g_{y}
\end{aligned}
$$


$\rho\left(\frac{\partial w}{\partial t}+u \frac{\partial w}{\partial x}+v \frac{\partial w}{\partial y}+w \frac{\partial w}{\partial z}\right)=-\frac{\partial P}{\partial z}+\eta\left(\frac{\partial^{2} w}{\partial x^{2}}+\frac{\partial^{2} w}{\partial y^{2}}+\frac{\partial^{2} w}{\partial z^{2}}\right)+\rho g_{z}$

where $\eta$ represents the viscosity [8], $\rho$ the fluid density, $P$ represents static pressure and $\vec{g}$ the gravitational acceleration. The energy equation specifies that the rate of a particle's energy change equals the sum of heat rate added to the fluid particles and the work made into the particle (Equation 3),

$$
\rho c_{p}\left(u \frac{\partial T}{\partial x}+v \frac{\partial T}{\partial y}+w \frac{\partial T}{\partial w}\right)=K\left(\frac{\partial^{2} T}{\partial x^{2}}+\frac{\partial^{2} T}{\partial y^{2}}+\frac{\partial^{2} T}{\partial z^{2}}\right)
$$

where $K$ represents the thermal conductivity, and $T$ the temperature. The viscosity was calculated from the is the shear stress to the strain rate ratio, $\eta=\tau / \dot{\gamma}$.

The solder motion in the wave was modeled by introducing the volume of fluid (VOF) for tracking the free surface. This scheme introduces a scalar quantity, $F$, that represents the fraction occupied in each computational cell by the solder. If $F=1$, the cell is fully occupied by solder; otherwise if $F=0$. A transport equation for $F$ is included in the form (Equation 4):

$$
\frac{\partial F}{\partial t}=u \frac{\partial F}{\partial x}+v \frac{\partial F}{\partial y}+w \frac{\partial F}{\partial w}
$$

Turbulence was modeled by the $k-\varepsilon$ model. This includes an equation for the transport of turbulent energy, $k$, (Equation 5 ) and another for the rate of dissipation, $\varepsilon$, (Equation 6):

$$
\begin{gathered}
\frac{\partial}{\partial t}(\rho k)+\frac{\partial}{\partial x_{i}}\left(\rho k u_{i}\right)= \\
\frac{\partial}{\partial x_{j}}\left[\left(\mu+\frac{\mu_{t}}{\sigma_{k}}\right) \frac{\partial k}{\partial x_{j}}\right]+G_{k}+G_{b}-\rho \varepsilon-Y_{M}+S_{k} \\
\frac{\partial}{\partial t}(\rho \varepsilon)+\frac{\partial}{\partial x_{i}}\left(\rho \varepsilon u_{i}\right)= \\
\frac{\partial}{\partial x_{j}}\left[\left(\mu+\frac{\mu_{t}}{\sigma_{\varepsilon}}\right) \frac{\partial \varepsilon}{\partial x_{j}}\right]+C_{1 \varepsilon} \frac{\varepsilon}{k}\left(G_{k}+C_{3 \varepsilon} G_{b}\right)-C_{2 \varepsilon} \rho \frac{\varepsilon^{2}}{k}+S_{\varepsilon}
\end{gathered}
$$

where $\mu_{t}$ represents the turbulent viscosity and $C_{x}$ are empirical constants [9].

The CFD simulation process was carried out using the ANSYSTM platform, Workbench ${ }^{\mathrm{TM}}$, for the meshing, solving and post-processing of the results. ANSYS ${ }^{\mathrm{TM}}$ provides the equation solver, Fluent $^{\mathrm{TM}}$ [10].

The discretization of the domain is based in the finite volume method, which consists in the division of the domain in small control volumes with constant properties. The solution algorithm scheme was the SIMPLE for the pressure-velocity coupling. The Standard method was used for the spatial discretization of the pressure while the Second Order Upwind was used for the momentum, $k, \varepsilon$ and energy equations. The simulation was executed in transient state, assuming constant the inputs.
The iterative method terminated when the residuals of continuity, energy and the $x, y$ and $z$ components of the momentum equations were less than $10^{-4}$. Table 1 summarizes the physical constants used for the solder.

Tab. 1: Physical constants

\begin{tabular}{|c|c|}
\hline Property & Value \\
\hline Surface tension & $0.548 \mathrm{~N} / \mathrm{m}$ \\
\hline Density & $7,290 \mathrm{~kg} / \mathrm{m}^{3}$ \\
\hline Viscosity & $0.015 \mathrm{~Pa} . \mathrm{s}$ \\
\hline Thermal Conductivity & $64 \mathrm{~W} / \mathrm{mK}$ \\
\hline
\end{tabular}

\section{RESULTS AND DISCUSSION}

The injection velocity from the solder jet that forms the wave was set at $1.05 \mathrm{~m} / \mathrm{s}$. This will provide a contact patch of approximately $20 \mathrm{~mm}$ across the PCB. At the free surface a constant pressure difference defined by Young and Lapalce's equation was included.

Figure 4 shows the interaction of the free jet with the PCB surface.

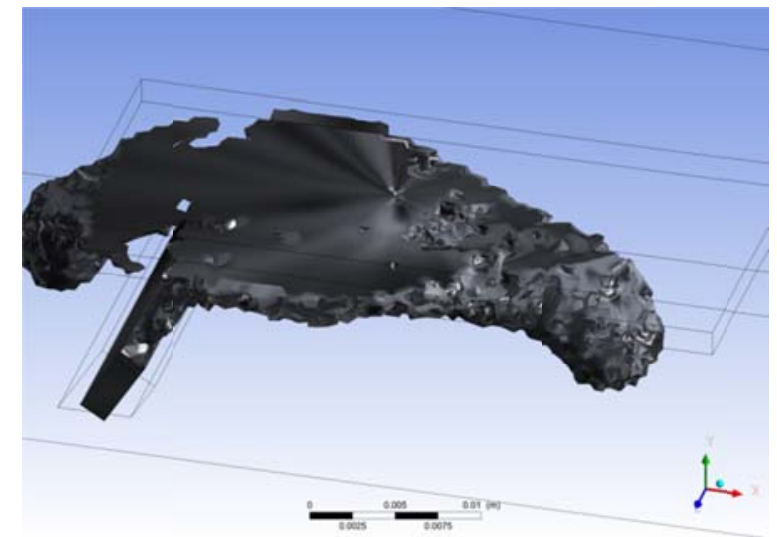

Fig. 4: View of the interaction of solder jet with the PCB.

The PCB was assumed on a horizontal position and the solder's nozzle at a $30^{\circ}$ angle with the vertical. The results show the formation of a jet dominated by inertia before gravity drives the flow downwards. It is also observed a backflow of some of the impinging fluid.

Figure 5 depicts the solder interaction just upstream of the component. The resulting pressure distribution is represented in Figure 6. Both the location of the jet impact on the PCB surface and the upstream face of the component show a higher static pressure. The highest, on the component's forward face, can reach 5,000 $\mathrm{Pa}$. 


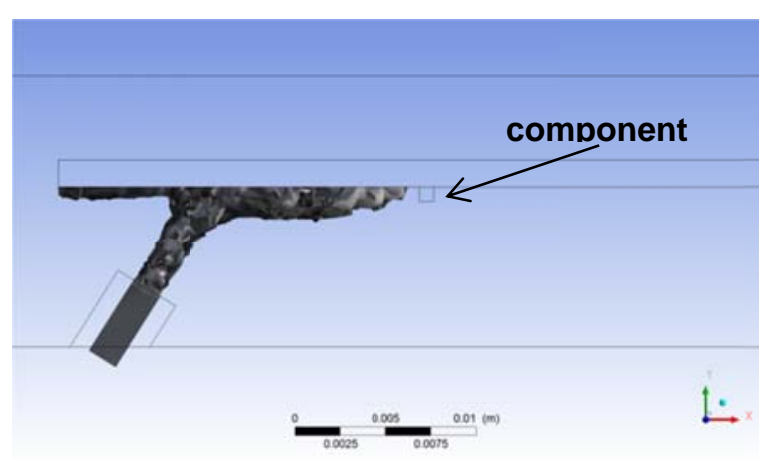

Fig. 5: Side view of the interaction of solder jet with the $\mathrm{PCB}$ and the electronic component.

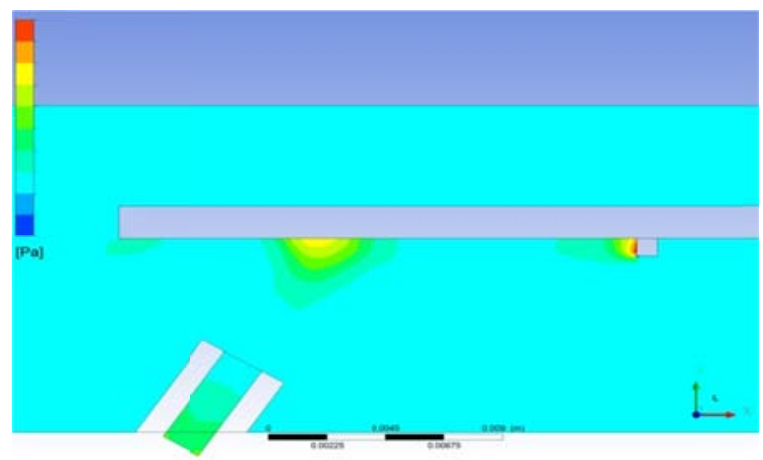

Fig. 6: Pressure field resulting from the interaction of a solder jet with the PCB and component.

From the computed pressure distribution along the surface one can calculate the total force that is applied to the component. In the present case this in the order of $0.2 \mathrm{~N}$. Preliminary results show that this value is in the same range as that measured experimentally.

Often the conveyor belt that drives the PCB through the soldering furnace is inclined at a small angle to the horizontal. Considering an angle of $8^{\circ}$, Figure 7 shows the dispersion of the solder on the PCB surface.

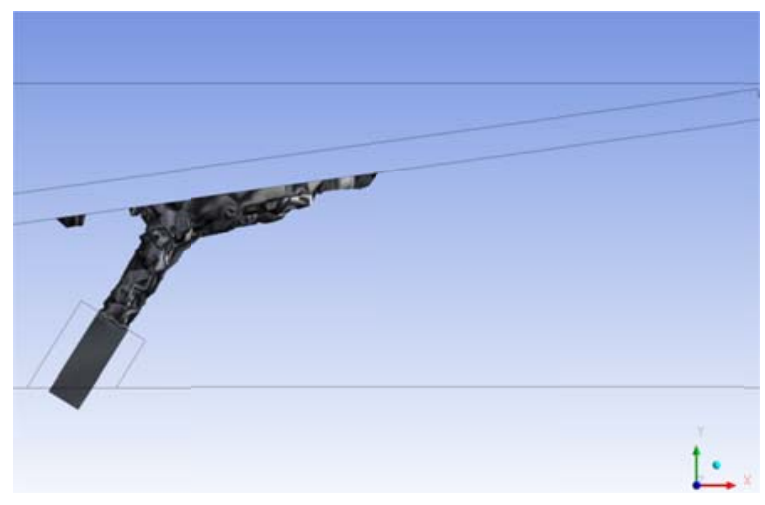

Fig. 7: Side view of the interaction of solder jet with the PCB and the electronic component. Elevation angle of $8^{\circ}$.
By changing the conveyor elevation angle a considerable reduction of the backflow is observed. The interaction of the wave with two components is shown in Figure 8.

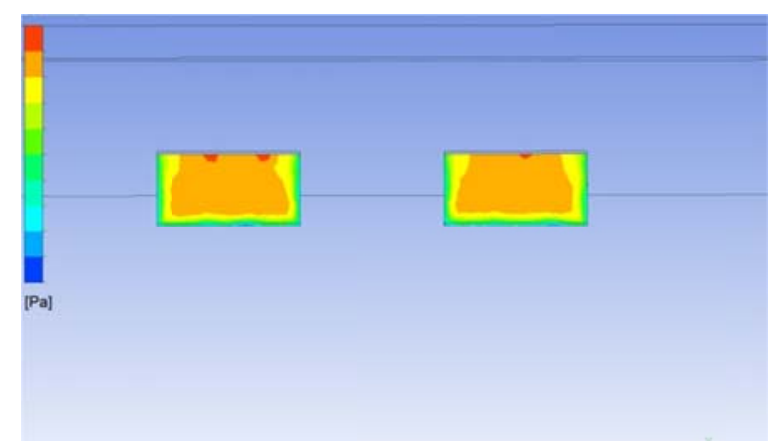

Fig. 8: Pressure distribution on the forward face of two electronic components resulting from the interaction with a solder jet.

The results show the static pressure distribution on the forward faces of the two components. The data shows that the pressure is evenly distributed on both components.

\section{EXPERIMENTAL VALIDATION}

In order to validate the drag forces on the component surface, an experimental apparatus has been designed and manufactured. At the moment only very preliminary data have been obtained and, therefore, only a description is presented here.

For this purpose, a blunt object (that will simulate an electronic component) is inserted onto the surface of a PCB. This has a small aperture that enables a metal beam (thickness of $0.5 \mathrm{~mm}$ ) to hold the blunt object. Therefore this item is not in direct contact with the $\mathrm{PCB}$ surface and so is free of constrainment. The beam has two strain gages, in opposite faces, that will monitor the bending stresses resulting from the solder wave interaction with the component. A second set of strain gages will be attached to identical beam that is not under mechanical stress. This is used to measure the thermal stress in the metal beam and this signal is taken as a zero reading, which is subtracted to the total stress measured by the other set of gages. In this way the influence of temperature is taken into account. The strain signals are processed by a HBM MGC Plus data recorder. Figure 9 depicts the experimental arrangement. The entire assembly is positioned into the soldering furnace by means of a set of screws. 


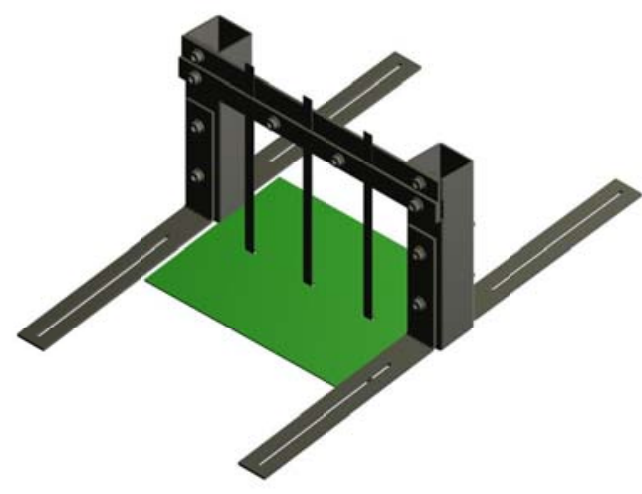

Fig. 9: View of the experimental arrangement for stress measurements.

\section{CONCLUSIONS}

The present work illustrates the application of CFD to describe the melted flow of solder in the wave soldering process of electronic components on the PCB surface.

The results show that modelling the wave interaction with the $\mathrm{PCB}$ and the various components can be used to compute the drag forces from the pressure distribution and, therefore, predict the occurrence of component disruption during the soldering process. The results also show that the force calculated is in agreement with the experimental evidence.

The model can be used to introduce various operating parameters such as the conveyor's velocity, the incidence angle and the physical properties.

\section{NOMENCLATURE}

CFD Computation Fluid Dynamics

PCB Printed Circuit Board

PTH Pin Through-Hole

$\vec{F} \quad$ Source term for the momentum equation

$F \quad$ Volume fraction

$\vec{g} \quad$ Gravitational acceleration

$k \quad$ Turbulent kinetic energy

$K \quad$ Thermal conductivity

$P \quad$ Pressure

$S_{h} \quad$ Source term for the energy equation

$T \quad$ Temperature

$u, v, w \quad$ Velocity components

$\varepsilon \quad$ Turbulent energy dissipation

$\dot{\gamma} \quad$ Strain rate

$\eta \quad$ Viscosity

$\rho \quad$ Density

$\tau \quad$ Stress tensor

\section{REFERENCES}

[1] Liukkonen, M; Havia, E; Leinonen, $\mathrm{H}$ and Hiltunen, $\mathrm{Y}$ (2009) Application of self-organizing maps in analysis of wave soldering process, Expert Systems With Applications, vol. 36, pp. 4604-4609.

[2] Abdul Aziz, M; Abdullah, M; Khor, C and Che Ani, F (2013) Influence of pin offset in PCB through-hole during wave soldering process: CFD Modeling approach, International Communications in Heat and MAss Transfer, vol. 48, pp. 116123.

[3] Liukkonen, M; Havia, E; Leinonen, H and Hiltunen, Y (2011) Quality-oriented optimization of wave soldering process by using self-organizing maps, Applied Soft Computing, vol. 11, pp. 214-220.

[4] Lee, NC (2002) Reflow Soldering Processes and Troubleshooting SMT, BGA, CSP, and Flip Chip Technologies, Boston: Butterworth Heinemann.

[5] Abdul Aziz, M; Abdullah, M; Khor, C; Jalar, A and Che Ani, F (2014) CFD modeling of pin shape effects on capillary flow during wave soldering, International Journal of Heat and Mass Transfer, vol. 72, pp. 400-410.

[6] Powell, R; Tan, W and Ume, I (2006) Experimental and Numerical Study of Effect of Plastic Ball Grid Array Package and Placement on PWB Warpage Convective Solder Reflow, in IMECE 2006-15678, Chicago, Illinois, USA.

[7] Shen, Y; Ng, C and Zheng, Y (2004) Simulation of wave propagation over a submerged bar using VOF method With a two-equation $k-\varepsilon$ turbulence modeling, Ocean Engineering, vol. 31, pp. 87-95.

[8] Khor, CY; Abdullah, MZ and Leong, WC (2012) Fluid/structure interaction analysis of the effects of solder bump shapes and input/output counts on moulded packaging, IEEE Trans. Components Packag. Techn. 2 (4) (2012) 604-616.

[9] Mompean, G (1998) Numerical Simulation of a Turbulent Flow Near a Right-Angled Corner Using The Speziale Non-Lienar Model With RNG k-E Equations, Computers and Fluids, vol. 27, pp. 847-859.

[10] ANSYS, 2011, ANSYS FLUENT Theory Guide, ANSYS Inc, Canonsburg, PA, USA. 